\title{
A patient in whom self-terminating ventricular fibrillation was a manifestation of myocardial reperfusion
}

\author{
Norbert $M$ van Hemel, J Herre Kingma
}

\begin{abstract}
Self-terminating ventricular fibrillation was recorded in a 47 year old woman without coronary artery or other structural heart disease. Reperfusion was thought to be responsible for the ventricular fibrillation because the arrhythmia started while the ST segment was returning to the baseline during an episode of silent ischaemia that was probably caused by coronary spasm. This case shows that potentially lethal arrhythmias can arise during reperfusion and that ventricular fibrillation during reperfusion may be self-terminating.
\end{abstract}

(Br Heart f 1993;69:568-571)

Transient occlusion of a major coronary vessel by spasm leading to subsequent ischaemia can cause serious ventricular arrhythmias. These ischaemia induced arrhythmias are clearly different from the reperfusion arrhythmia that is caused by the re-establishment of coronary flow to the ischaemic region after transient occlusion. ${ }^{1-3}$ Occlusion arrhythmias are associated with progressive ST segment deviation whereas reperfusion arrhythmias occur when the ST segment is returning to baseline. ${ }^{1-3}$ We describe an unusual patient in whom self-terminating ventricular fibrillation (VF) was the only manifestation of reperfusion after coronary artery spasm.

\section{Case report}

On 4 April 1990 a 47 year old woman was admitted to the emergency room after a sudden cardiac arrest at rest without any previous signs or symptoms. Her husband had started cardiopulmonary resuscitation immediately. The paramedical staff on the ambulance, which arrived within eight minutes, recorded ventricular fibrillation and restored sinus rhythm with two $400 \mathrm{DC}$ shocks. The circulation was stabilised within minutes. Apart from the cardiac arrest the patient did not have any other cardiac symptoms. Her previous medical history was unremarkable and there was no family history of cardiovascular disease. She had smoked 25 cigarettes a day for many years. Physical examination showed no abnormalities. Blood pressure was $130 / 80 \mathrm{~mm} \mathrm{Hg}$. After admission to the coronary care unit an acute myocardial infarction was excluded on the basis of electrocardiography, serum creatine kinase concentrations, and cross sectional echocardiography. $\frac{0}{0}$ Additional laboratory examination showed a $\frac{\bar{\sigma}}{\bar{c}}$ normal lipid pattern (serum cholesterol 4.8 , $\overparen{\varnothing}$ high density lipoprotein cholesterol $1 \cdot 23, \cong$ triglycerides $1.24 \mathrm{mmol} / \mathrm{l}$ ) and the results of $\mathrm{a}^{\mathrm{e}}$ routine haematological and blood chemistry $\overrightarrow{0}$ were normal. During the first day after $\overrightarrow{\vec{\omega}}$ admission she showed signs of minor $\stackrel{\omega}{\sigma}$ ischaemic brain damage but these disappeared within a week.

Graded bicycle stress testing carried out 14 के days after cardiac arrest showed a maximal or exercise tolerance of eight minutes $(105 \mathrm{~W}) \varnothing$ without symptoms. At maximal heart rate the ST segment changes of the 12 lead electro- $\overrightarrow{ }$ cardiogram suggested ischaemia (fig 1): pre- $£$ mature ventricular extrasystoles were seen ${ }^{\oplus}$ only in the recovery phase. This ischaemic $\vec{\theta}$ pattern disappeared within five minutes after $\omega$ the maximal workload was achieved. She did $\square$ not complain of chest pain during stress testing. Right and left heart catheterisation did $\frac{D}{0}$ not show any abnormalities. Coronary arteriography showed normal arteries and there $\stackrel{\varnothing}{\AA}$ was no evidence of bridging segments. Supine $\overrightarrow{\vec{F}}$ bicycle graded exercise testing carried out $\frac{0}{3}$ during the invasive study did not provoke coronary spasm. Ergometrine $(0.1$ and 0.2 . $\mathrm{mg}$ ) given every five minutes during coronary arteriography did not provoke any ST segment changes, coronary spasm, or ventricular arrhythmia. ${ }^{4}$

Continuous two channel Holter recording when she was off treatment showed several $₹$ episodes of symptom-free ST segment 0 changes (maximum deviation $3 \mathrm{~mm}$ ). There was a diurnal pattern with profound ischaemic episodes being more common in $\mathrm{N}$ the morning (8.00-9.00 am) and night $(8.00-9.00 \mathrm{pm})$, as was reported in patients 0 with angina. ${ }^{35}$ The increase in sinus rate that $\omega$ preceded ST segment deviation was not remarkable but sinus tachycardia up to 140 beats/min was frequently recorded at the time $\mathbb{\infty}$ of peak ST segment deviation. The recording on 21 April 1990 showed an asymptomatic episode of profound ST segment change at 2016 . This was followed by recovery of the $\mathbb{\nabla}$ ST segment and decrease in sinus rate to the baseline value (fig 2). Without warning arrhythmias, ventricular fibrillation started $\delta$ four minutes after peak ST segment changes, when abnormal $T$ waves were the only residual signs of silent ischaemia. Ventricular fibrillation causing syncope persisted for 81 seconds, then organised to become a ventricular flutter ( 300 beats $/ \mathrm{min}$ ) with a torsades de pointes pattern which lasted 17 seconds. This 
Figure 1

Electrocardiographic

changes during graded exercise testing. Leads I to III,

aVR to $a V F$ are shown in

panels $A, B, C$, and leads

$V 1$ to V6 in $D, E, F$.

Panels $A$ and $D$ display the resting 12 lead ECG pre-

ceding stress

testing. Biphasic P waves

in leads II and III suggest

an abnormal site of

supraventricular impulse

formation. Slight ST seg-

ment elevation in leads II,

III, aVF, V2 to V6 may be

categorised as "early repo-

larisation". Panels B and

E show abnormal ST seg-

ments at maximally

achieved heart rate of 180

min $^{-1}$ (100\% of predicted

value). ST segment depres-

sion suggesting ischaemia is

clearly visible in leads II,

aVF, V3 to V6, while ST

segment elevation is present

in aVR. Note the appear-

ance of normal $P$ waves

during exercise. Panels $C$

and $F$ display absence of

ischaemic ST segments dur-

ing exercise during treat-

ment with calcium

antagonists at a maximal

heart rate of $180 \mathrm{~min}^{-1}$.

Figure 2 Holter recording showing self-limiting ventricular fibrillation. This one lead recording (paper speed $6.5 \mathrm{~mm} / \mathrm{s}$ ) shows the development of gradual and profound $S T$ depression $(8.16 \mathrm{pm})$ and the return of the ST segment to the baseline $(8.21 \mathrm{pm})$ and finally residual negative $T$ waves. Without preceding arrhythmias ventricular fibrillation emerged and terminated spontaneously after a phase of ventricular flutter with a torsades de pointes pattern. Finally sinus rhythm resumed after a long sinus arrest and an episode of transient atrioventricular block.

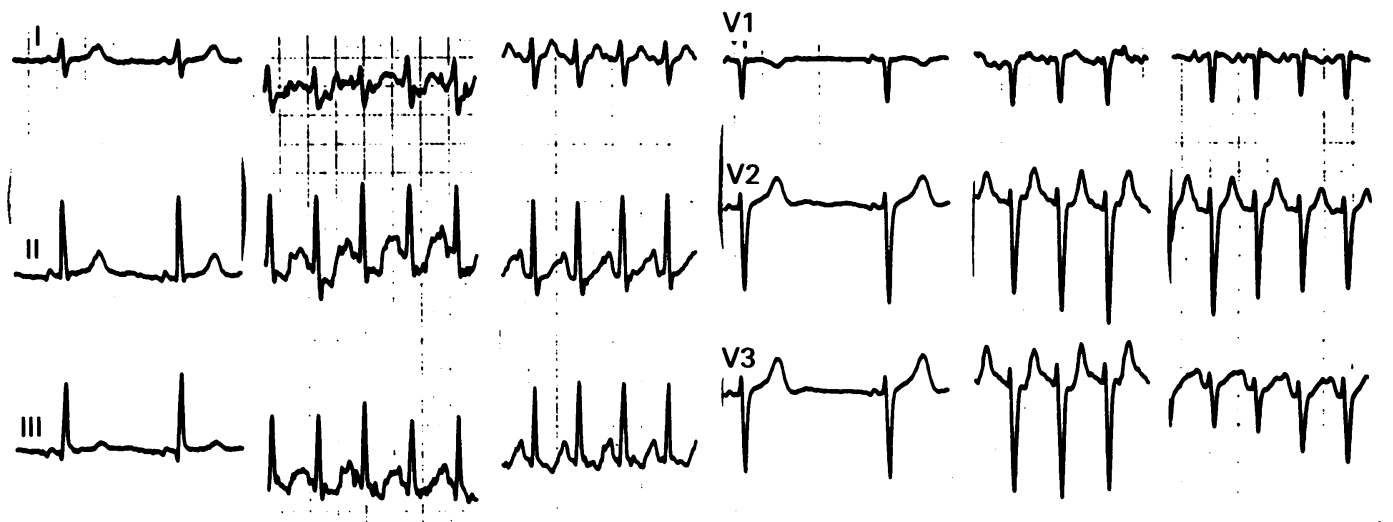

(n)

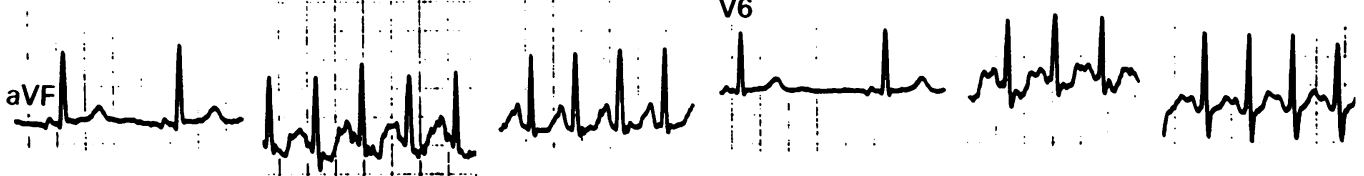
A

B

C

D

E

F

HR

$\left(\mathrm{min}^{-1}\right)$

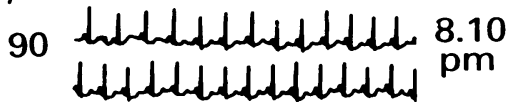

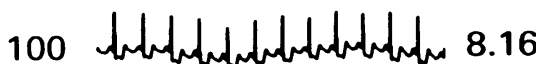

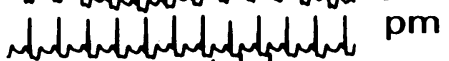

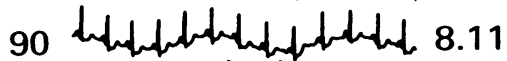

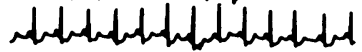

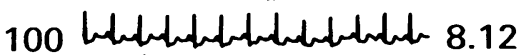

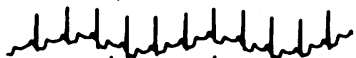

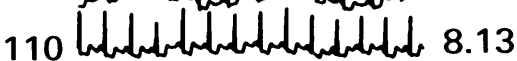

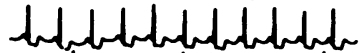

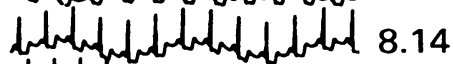

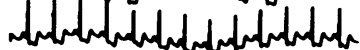

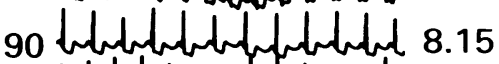

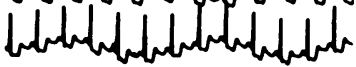

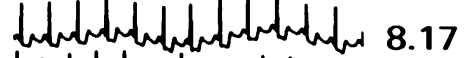

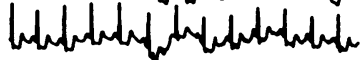

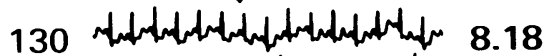

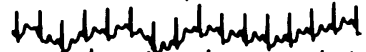

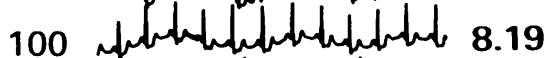

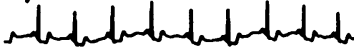

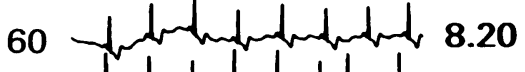

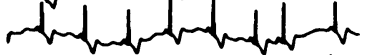

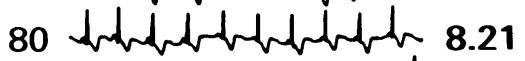
butrobuthert

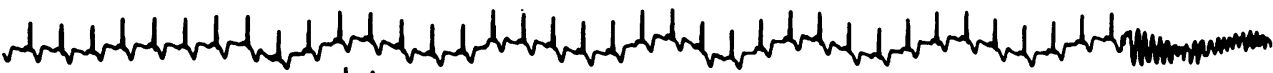
8.22

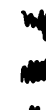

8.24

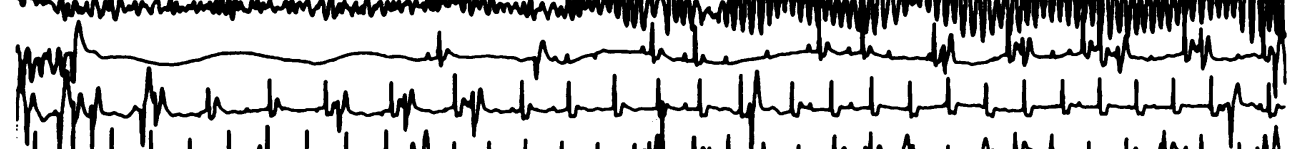

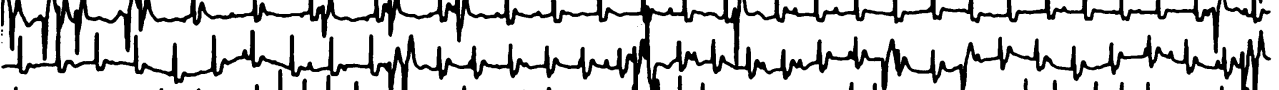

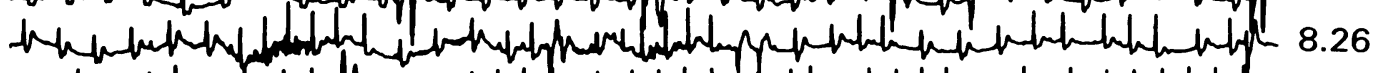

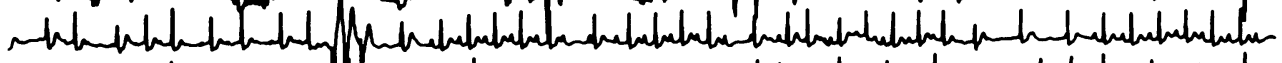

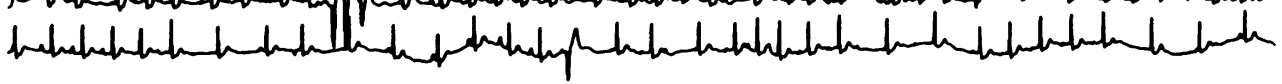


arrhythmia terminated spontaneously and was followed by long sinus pauses until, after a transient third atrioventricular block, normal sinus rhythm resumed. After she recovered consciousness the patient cleaned up urine in her room before she asked for help from the nursing staff, who only then became aware of this serious cardiac event.

The most likely diagnosis was selfterminating ventricular fibrillation caused by asymptomatic ischaemia that was precipitated by coronary spasm. Treatment with calcium antagonists was given to prevent coronary spasm. Successive Holter recordings and bicycle stress tests (fig 1) showed no new attacks of ischaemia, coronary spasm, or ventricular arrhythmias. After a two year of follow up the patient is still taking nifedipine (80 $\mathrm{mg}$ daily), and lives a normal life without symptomatic arrhythmia, dizziness, or syncope.

\section{Discussion}

Spasm of coronary arteries exposes the myocardium to the double hazard of transient ischaemia and subsequent reperfusion. Postmortem studies in cases of sudden cardiac death showed minor recent coronary thrombi or plaque fissuring causing $<50 \%$ luminal occlusion in half of those examined. ${ }^{6}$ In the remainder, lethal arrhythmias were a possible cause of sudden death. ${ }^{7}$ In those without obstructive coronary artery disease coronary spasm is likely to be important in inducing ischaemia and the associated subsequent reperfusion. Few patients without coronary lesions showed definite evidence of coronary spasm. ${ }^{8-12}$ Ventricular fibrillation and sudden cardiac death, however, have been reported in this category of patients, ${ }^{8-11}$ sometimes without preceeding chest pain ${ }^{12}$ as in our patient.

Ventricular arrhythmias caused by coronary spasm in patients with Prinzmetal variant angina were more common during coronary occlusion than during reperfusion of the ischaemic myocardium. ${ }^{124}$ Positive correlations were reported between the incidence of ventricular arrhythmias during reperfusion and the duration of myocardial ischaemia and the degree of ST segment changes. ${ }^{12}$ Such correlation was also found in Langendorf ischaemic-reperfusion rat hearts. ${ }^{13}$ Furthermore, the risk of reperfusion arrhythmias in Prinzmetal variant angina seems inversely related to the degree of coronary artery disease. ${ }^{14}$ This suggests that normal myocardium without previous scarring and with a normal oxygen consumption is a prerequisite for the initiation of ventricular arrhythmias by reperfusion.

Recent experimental studies have shed light on the arrhythmogenic mechanisms that are responsible for reperfusion ventricular arrhythmias. Priori et al showed the emergence of early afterdepolarisations which trigger automated activity in reperfused ischaemic myocardium. ${ }^{15}$ However, early afterdepolarisations can also prolong the refractory periods in a nonhomogeneous manner which enhances the dispersion of refractory periods and the facilitation of reentrant arrhythmias. ${ }^{16}$ Abnormal responses to $\alpha$ and $\beta$ adrenergic receptor stimulation ${ }^{17}$ and superoxide radical formation during ischaemia ${ }^{18}$ could also influence arrhythmogenesis.

Though coronary artery spasms could not be provoked in our patient, who did not have coronary atherosclerosis, the Holter recording showed several painless ischaemic episodes suggestive of coronary spasm. Ventricular fibrillation started at the time the spasm was relieved (shown by normalisation of the ST segment) and reperfusion occurred. Tzivoni et $a l^{11}$ described reperfusion ventricular fibrillation in a patient without coronary artery disease, but VF was preceded by chest pain and direct current shock was immediately applied. Crozier $e t a l^{12}$ reported on the resuscitation of two patients with ventricular fibrillation and ventricular asystole, respectively, without preceding chest pain. Coronary arteriography showed normal arteries in both patients. It is not clear whether ischaemia due to occlusion or reperfusion played a part in these cases. These observations show that symptomatic and life-threatening ventricular arrhythmias are much rarer after episodes of coronary spasm than after symptomatic ischaemia. $^{3}$

Self-terminating VF is unusual; presumably it occurs only when there is rapid and complete re-establishment of prexisting normal electrophysiological properties in the myocardium. Recently Kontny and Dale reported on a case of self-terminating VF in a patient without apparent organic heart disease. ${ }^{19}$ We found no structural cardiac abnormalities in our patient, and the spontaneous termination of VF was probably the result of rapid electrophysiological improvement during the dynamic process of reperfusion. Undoubtedly this is why the configuration of VF became organised into ventricular flutter with subsequent spontaneous restoration of sinus rhythm. In view of this arrhythmogenic mechanism we used nifedipine to prevent the recurrence of $\mathrm{VF}^{12}$ because calcium antagonists can suppress coronary spasms and possibly also reperfusion arrhythmias. ${ }^{2021} \mathrm{We}$ found no reason for the ischaemic ST segment pattern seen during the first exercise test after VF. Apart from this pattern there was no other evidence of an insufficient myocardial oxygen supply. Because this pattern could not be reproduced at later exercise testing we presume that it was a transient metabolic disorder of the myocardium caused by the arrhythmic event.

In patients without structural heart disease the cause of sudden cardiac death remains unknown: intense functional changes alone are held to be responsible for lethal arrhythmic events. ${ }^{7}$ In our patient reperfusion after asymptomatic ischaemia caused by coronary spasm could have triggered this episode of VF. The self-termination of VF was remarkable and presumably it was related to reperfusion itself, because there was no pre-existing 
structural myocardial abnormality. In addition, this study emphasises the benefits of long-term ambulatory electrocardiographic recordings to detect ST segment changes in patients with unexplained syncope or socalled idiopathic ventricular arrhythmias.

1 Previtali M, Klersy C, Salerno JA, et al. Ventricular tachyarrhythmias in Prinzmetal's variant angina: Clinical sig nificance and relation to the degree and time course of S-T segment elevation. $A m$ f Cardiol 1983;52:19-25.

2 Kerin NZ, Rubenfire M, Willens J, Rao P, Cascade PN The mechanism of dysrhythmias in variant angina pectoris: Occlusive versus reperfusion. Am Heart $\mathcal{f} 1983$; 6:1332-40.

3 Araki H, Koiwaya Y, Nakagaki O, Nakamura M. Diurna distribution of ST-segment elevation and related arrhythmias in patients with variant angina: A study by ambulatory ECG monitoring. Circulation 1983;67: 995-1000.

4 Szlachcic J, Waters DD, Miller D, Théroux P. Ventricular arrhythmias during ergonovine-induced episodes of variant an dur

5 Deedwania PC, Nelson JR. Pathophysiology of silent myocardial ischemia during daily life. Hemodynamic myocardial ischemia during daily life. Hemodynamic blood pressure monitoring. Circulation 1990;82. blood press

6 Davies MJ, Thomas A. Thrombosis and acute coronary artery lesions in sudden cardiac ischemic death. $N$ Engl F Med 1984:310:1137 40 .

7 Myerburg RJ, Kessler KM, Bassett AL, Castellanos A. A biological approach to sudden cardiac death: structure, function and cause. Am $\mathcal{F}$ Cardiol 1989;63:1512-6.

8 Maseri A, Severi S, Marzullo P. Role of coronary arterial spasm in sudden coronary ischemic death. In Greenberg HM, Dwyer EM, eds. Sudden coronary death. New York: New York Academy of Sciences, 1982;382,204-17.

9 Puddu PE, Bourassa MG, Waters DD, Lesperance J. Sudden death in two patients with variant angina and apparently minimal fixed coronary stenosis and Electrocardiol 1983;16:213-20.
10 Conti RC, Pepine CJ, Curry RC. Coronary artery spasm: an important mechanism in the pathology of ischemic heart disease. In: Proctor Harvey W, ed. Current problems in cardiology. Chicago and London: Year Book Medical Publishers 1979;4:1.

11 Tzivoni D, Keren A, Granot H, Gottlieb S, Benhorin J, Stern $S$. Ventricular fibrillation caused by myocardial reperfusion in Prinzmetal's angina. Am Heart f 1983; 105:323-5.

12 Crozier I, Stewart D, Ikram H. Sudden death due to painless spasm in near normal coronary arteries. Aust $N Z \mathcal{F}$ Med 1986;16:64-5.

13 van Gilst WH, de Graeff PA, Kingma JH, Wesseling $\mathbf{H}$, de Langen CDJ. Captopril reduces purine loss and reperfusion arrhythmias in the rat heart after coronary reperfusion arrhythmias in the rat heart af

14 Sheehan FH, Epstein SE. Determinants of arrhythmic death due to coronary spasm: effects of preexisting coronary artery stenosis on the incidence of reperfusion rrhythmia. Circulation 1982;65:259-64.

15 Priori SG, Mantica M, Napolitano C, Schwartz PJ. Early afterdepolarizations induced in vivo reperfusion of ischemic myocardium. A possible mechanism for reperfusion arrhythmias. Circulation 1990;81:1911-20.

16 Pogwizd SM, Corr PB. Electrophysiologic mechanisms underlying arrhythmias due to reperfusion of ischemic myocardium. Circulation 1987;72:404-26.

17 Sheridan DJ, Penkoskic PA, Sobel BE, Corr PB. Alphaadrenergic contributions to dysrhythmia during myocardial ischemia and reperfusion in cats. $\mathcal{F}$ Clin Invest 1980; 65:161-71.

18 Manning AS, Coltrat DJ, Hearse DJ. Ischemia and reperfusion-induced arrhythmias in the rat: effects of xanfusion-induced arrhythmias in the rat: effects of xan-
thine oxidase inhibition with allopurinol. Circ Res 1984; 55:545-8.

19 Kontny F, Dale J. Self-terminating idiopathic ventricular fibrillation presenting as syncope: a 40 -year follow-up report. F Intern Med 1990;227:211-3.

20 Swies J, Omogbai EKI, Smith GM. Occlusion and reperfusion induced arrhythmias in rats: involvement of platelets and effects of calcium antagonists. $\mathcal{F}$ Cardiovasc Pharmacol 1990;15:816-25.

21 van Gilst WH, Kingma JH, Terpstra JA, Viersma JW, Wesseling $H$. Post-exercise ventricular arrhythmias reduced by the calcium entry blocker, diltiazem [abstr] f Am Coll Cardiol 1987;9:228. 